\section{Evaluación de docentes clínicos de Postgrado: desarrollo y propiedades psicométricas del instrumento MEDUC-PG14}

\author{
MARGARITA PIZARRO ${ }^{1, \mathrm{a}}$, NANCY SOLÍS ${ }^{1, \mathrm{a}}$, VIVIANA ROJAS ${ }^{1, \mathrm{~b}}$, \\ LUIS ANTONIO DÍAZ ${ }^{2}$, OSLANDO PADILLA ${ }^{3, \mathrm{c}}$, LUZ MARÍA LETELIER ${ }^{2,6}$, \\ ANDRÉS AIZMAN ${ }^{2,6}$, ALBERTO SARFATIS ${ }^{2}$, TRINIDAD OLIVOS ${ }^{4, b}$, \\ ALEJANDRO SOZA ${ }^{1}$, ALEJANDRO DELFINO ${ }^{5}$, GONZALO LATORRE $^{2}$, \\ DANISA IVANOVIC-ZUVIC ${ }^{2}$, TRINIDAD HOYL ${ }^{2,6}$, MARCELA BITRAN ${ }^{7, a}$, \\ JUAN PABLO ARAB ${ }^{1}$, ARNOLDO RIQUELME ${ }^{1,7}$
}

\section{Development of MEDUC-PG14 survey to assess postgraduate teaching in medical specialties}

Background: Feedback is one of the most important tools to improve teaching in medical education. Aim: To develop an instrument to assess the performance of clinical postgraduate teachers in medical specialties. Material and Methods: A qualitative methodology consisting in interviews and focus-groups followed by a quantitative methodology to generate consensus, was employed. After generating the instrument, psychometric tests were performed to assess the construct validity (factor analysis) and reliability (Cronbach's alpha). Results: Experts in medical education, teachers and residents of a medical school participated in interviews and focus groups. With this information, 26 categories (79 items) were proposed and reduced to 14 items (Likert scale 1-5) by an expert's Delphi panel, generating the MEDUC-PG14 survey, which was answered by 123 residents from different programs of medical specialties. Construct validity was carried out. Factor analysis showed three domains: Teaching and evaluation, respectful behavior towards patients and health care team, and providing feedback. The global score was $4.46 \pm 0.94$ (89\% of the maximum). One teachers' strength, as evaluated by their residents was "respectful behavior" with $4.85 \pm 0.42$ (97\% of the maximum). "Providing feedback" obtained $4.09 \pm 1.0$ points $(81.8 \%$ of the maximum). MEDUC-PG14 survey had a Cronbach's alpha coefficient of 0.947. Conclusions: MEDUC-PG14 survey is a useful and reliable guide for teacher evaluation in medical specialty programs. Also provides feedback to improve educational skills of postgraduate clinical teachers.

(Rev Med Chile 2015; 143: 1005-1014)

Key words: Educational measurement; Questionnaires; Specialties, medical.

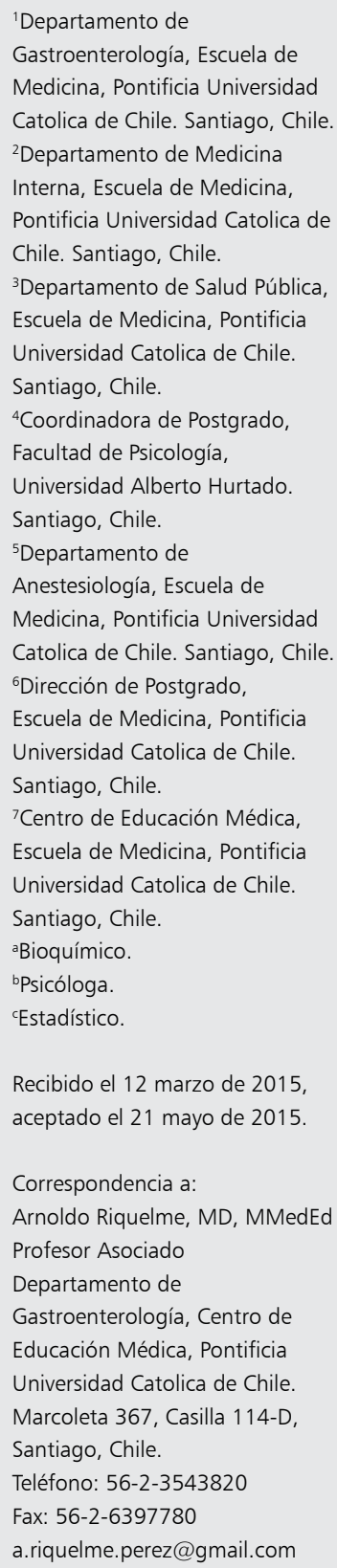

Recibido el 12 marzo de 2015 , aceptado el 21 mayo de 2015 .

Correspondencia a: Arnoldo Riquelme, MD, MMedEd Profesor Asociado Departamento de Gastroenterología, Centro de Educación Médica, Pontificia Universidad Catolica de Chile. Marcoleta 367, Casilla 114-D Santiago, Chile.

Teléfono: 56-2-3543820

Fax: 56-2-6397780

a.riquelme.perez@gmail.com

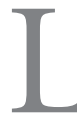

os programas de postgrado en medicina han alcanzado un gran desarrollo en los últimos años, gracias al advenimiento de nuevas tecnologías y la necesidad de mayor conocimiento específico en el manejo de enfermedades complejas, las que exigen progresivamente mayor entrenamiento y trabajo en equipo ${ }^{1}$. El desarrollo de estos programas se basa en la capacidad de transmisión de conocimientos, destrezas y actitudes por parte de especialistas (docentes), 
los cuales son altamente calificados en el manejo de pacientes, pero que, en general, no tienen una formación profesional en aspectos relacionados con la docencia ${ }^{2}$.

La mayoría de los programas buscan mejorar la metodología de enseñanza y la implementación de instrumentos de evaluación ${ }^{3}$. Otros aspectos de la enseñanza menos desarrollados por los docentes incluyen: habilidades comunicacionales, diseño curricular, identificación y desarrollo de competencias médicas o aquellas relacionadas con el inter-profesionalismo ${ }^{4}$. En la actualidad, estas falencias han otorgado mayor protagonismo a la capacitación docente, de hecho, estos programas deben transmitir la metodología adecuada para educar a residentes en todos los aspectos del quehacer médico y no sólo en habilidades clínicas 5 . Todos estos puntos forman parte de conceptos innovadores en docencia, los que están en sintonía con los cambios curriculares en programas de postítulo.

Por otra parte, un aspecto de vital importancia en el proceso de desarrollo e implementación de un programa es la evaluación docente. Esta última es más que un mero ejercicio académico, ya que los resultados que emanen de ella servirán para futuros cambios en el proceso de selección y capacitación docente de cada institución, con especial impacto en los formatos de entrega de conocimientos que promuevan un proceso de enseñanza y aprendizaje más eficaz ${ }^{6}$. En efecto, Steinert estableció, hace más de 10 años, que la capacitación docente debe ser la guía que promueva los procesos educativos de una institución y debe cumplir con un rol de modelaje de todo lo que se hace al interior de ésta ${ }^{7}$.

Por otra parte, la docencia no es sólo entregar información, sino que involucra un complejo proceso que requiere apoyo constante a los profesores clínicos $^{8}$. En este sentido, la retroalimentación constituye una de las herramientas más importantes para mejorar la docencia y el aprendizaje en educación médica-11 ${ }^{9}$, por lo que un programa de excelencia debería buscarla activamente con diversos recursos.

Dada la importancia de contar con un método de evaluación, se han desarrollado diversos instrumentos con este fin, que consideran las habilidades descritas previamente. Entre ellos destacan el Maastricht Clinical Teaching Questionnaire (MCTQ) ${ }^{12}$, considerado de gran rigu- rosidad metodológica y orientado a la docencia hospitalaria, el instrumento Medicina Universidad Católica 30-ítems (MEDUC-30) ${ }^{13}$, para evaluar el desempeño docente en profesores de pregrado y el Medicina Universidad Católica, Radiología 32-items (MEDUC-RX32) ${ }^{14}$, para evaluar la enseñanza clínica en profesores de radiología, ambos desarrollados en la Escuela de Medicina de la Pontificia Universidad Católica de Chile (EMPUC). También existen otras herramientas como el Student Evaluation of Teaching in Outpatient Clinics (SETOC), específicamente desarrollado para la evaluar el desempeño docente en educación en postgrado ambulatorio ${ }^{15}$, el cuestionario Stanford Faculty Development Program (SFDP26) ${ }^{16}$ y la University of Michigan Global Rating Scale (GRS) ${ }^{17}$.

En postgrado, la docencia clínica tutorial tiene lugar en múltiples contextos clínicos, de acuerdo a cada especialidad y durante esta interacción docente-residente, el profesor puede recibir retroalimentación de su desempeño. Sin embargo, no existen instrumentos validados a nivel latinoamericano para la evaluación docente.

El objetivo del presente estudio fue el desarrollo y validación de un instrumento de Evaluación del Desempeño de Docentes Clínicos de Postgrado en Medicina. Dicho instrumento debe ser válido para variados contextos e instancias de docencia, incluyendo ambientes ambulatorios y hospitalarios, programas quirúrgicos y no quirúrgicos y cuyos resultados sirvan como indicador de la calidad de enseñanza recibida de parte de los profesores clínicos de postgrado y de los programas de especialidades médicas a los cuales pertenecen.

\section{Métodos}

\section{Desarrollo del instrumento}

El estudio consideró una metodología mixta, incluyendo una fase cualitativa y otra cuantitativa. Se realizó una entrevista individual semi-estructurada a un representante de la Agencia Acreditadora de Programas de Postgrado, de Especialidad en Medicina y de Centros Formadores de Especialistas Médicos (APICE). Además, se llevó a cabo un grupo focal que consideró a 8 jefes de programas de postgrado en medicina de la Facultad de Medicina de la EMPUC, los que fueron invitados en forma personal, considerando un muestreo aleatorio estratificado que garantizara la presen- 
cia de representantes de varios de los diferentes programas vigentes en el grupo. El procedimiento contó con la aprobación del comité de ética de la EMPUC y cada participante firmó un consentimiento informado. Se empleó el análisis de contenido para llegar a conformar las categorías, apoyándose en elementos basados en la teoría fundada ${ }^{18}$. También se realizó un levantamiento de información en relación a un estudio de ambiente educacional en residentes utilizando la técnica de "seguir la mecha"19. Esta última consiste en la evaluación iterativa de un conjunto de datos, donde se analizan "aspectos claves" en un levantamiento de información, que inicialmente fue realizado con otro fin, para obtener nuevos datos ${ }^{20}$. Posteriormente se trianguló la información así obtenida, para luego conformar categorías con el software computacional ATLAS ti. La información recogida se trianguló con la participación de tres analistas y se consideró saturada tras la revisión exhaustiva del material extraído de las fuentes mencionadas. A partir de estos datos, se generaron los ítems que constituyeron el instrumento, el que finalmente se refinó mediante un panel de expertos con la técnica Delfi modificada.

\section{Participantes y aplicación}

$\mathrm{El}$ instrumento fue piloteado en residentes de 12 especialidades médicas de la EMPUC durante el período diciembre de 2014 a enero de 2015. Fue aprobado por el comité de ética de nuestra institución y se realizó un consentimiento informado por cada participante; además, los datos fueron entregados y utilizados en forma anónima.

\section{Análisis estadístico}

Se evaluó la validez de constructo mediante un análisis factorial exploratorio con rotación Varimax. Los factores se seleccionaron de acuerdo al criterio de Kaiser-Guttman, que incluye a los factores con un auto-valor (eigenvalue) > $1^{23-24}$. También se utilizó el criterio de Cattell, que establece el punto de inflexión del gráfico de sedimentación como valor de corte, considerando todos los factores sobre éste ${ }^{24}$.

La confiabilidad del instrumento fue medida mediante el coeficiente alfa de Cronbach ${ }^{25}$ y se consideró un valor confiable $\geq 0,7$. También se realizaron análisis estadísticos generales (media y desviación estándar). Para todos los análisis se utilizó el software computacional SPSS v15.0.

\section{Resultados}

\section{Desarrollo del instrumento}

Se obtuvo información a partir de una entrevista individual a un representante de APICE y un grupo focal que consideró 8 jefes de programas de postgrado en medicina de la EMPUC. Las especialidades médicas presentes en el grupo, en orden alfabético, fueron: Cirugía Digestiva, Dermatología, Enfermedades Respiratorias del Niño, Geriatría, Hematología, Infectología Pediátrica y Oncología. También se realizó un levantamiento de información en relación a un estudio de ambiente educacional en 8 grupos focales de residentes de programas de postgrado, el cual fue analizado para conocer aspectos vinculados a la didáctica en una tesis de título de psicología educacional. Posteriormente se trianguló la información de los residentes con el levantamiento obtenido en la entrevista individual y el grupo focales, que corresponden a la perspectiva de los acreditadores de programas y de los docentes, respectivamente ${ }^{19-20,27}$ (Figura 1). Se realizó una categorización abierta de los conceptos obtenidos, a partir de la cual se desarrollaron 26 categorías (Tabla 1).

Luego de la triangulación, se obtuvieron 109 ítems que posteriormente se redujeron a 79, al fusionar ítems y eliminar conceptos repetidos. Posteriormente, la Dirección de Postgrado de la EMPUC determinó cuales eran las necesidades principales a evaluar de sus profesores, las que se unieron junto a los 79 ítems conseguidos para obtener el instrumento final de 14 ítems (escala 1 a 5) (Figura 1). Además, se decidió agregar una nota de evaluación global (escala 1 a 7 ) al final de la pauta, ya que estudios previos sugieren que ésta tiene buena sensibilidad para identificar a los mejores docentes ${ }^{15}$.

Finalmente, un panel de expertos refinó la redacción de los ítems mediante técnica Delfi modificada, con la participación de 6 jefes de programa más 9 colaboradores y expertos en educación médica. Se realizó un consenso (escala Likert 1-5). Aquellos ítems con puntaje $<4$ fueron refinados o modificados para evitar ambigüedad. Se consideró como redacción definitiva aquellos ítems con grado de acuerdo $\geq 4$.

\section{Estadística general}

Los residentes respondieron 123 encuestas correspondientes a 79 docentes, que a su vez per- 


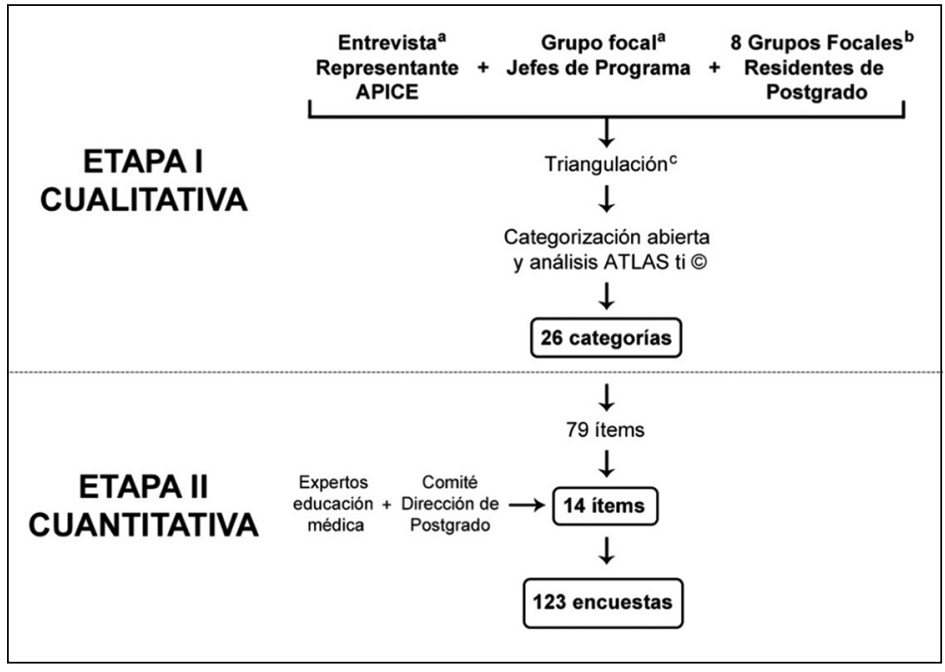

Figura 1. Diagrama de flujo del desarrollo del instrumento MEDUC-PG14. ${ }^{\text {TTeoría }}$ Fundada17. ${ }^{\text {T} T e ́ c n i c a ~ " S e g u i r ~ l a ~ m e c h a " 19,20 . ~}$ 'Triangulación de los datos ${ }^{21}$.

\section{Tabla 1. Categorías generadas a partir del levantamiento de información y sus citas verbales más} representativas

\section{Categorías Citas}

1. Promueve la Proactividad - "lo que uno más debe enseñar es cómo aprender"

- "estimular en ellos un constante deseo y necesidad de aprendizaje"

2. Establece comunicación fluida con los distintos actores del sistema de salud

- "la enfermera con su equipo son los que le facilitan la vida al enfermo, las que perciben oportunamente al dolor... entonces esa gente es fundamental, y la relación que tiene el residente con esas personas tiene que ser de muy buena calidad" con quienes se relaciona

3. Promueve actuación profesional ética en los comportamientos cotidianos

4. Transmite conciencia y valor - "es súper importante porque nosotros somos muy multidisciplinarios" de la interdisciplinariedad en el ejercicio de la medicina

- "el percibir que existe un problema ético necesita de un entrenamiento especial del supervisor que tiene que transmitírselo al supervisado"

Colabora con residentes en períodos de mayor presión asistencial

- "es indudable que cuando los residentes están en período junio, julio... la presencia de un buen supervisor con experiencia clínica y con capacidad, va a aliviar enormemente el trabajo porque se va a dar cuenta más rápido de quienes están más graves"

- " a veces les cargamos muchísimo la mano a los residentes y perdemos el norte para formarse de buena manera"

6. Entrega feedback estructura- - "feedback que no es solamente sentarse cada tres meses con el becado a reconocer do (retroalimentación) errores o favorecer cosas buena sino que es en el día a día"

- "tiene que ser muy objetivo, no puede ser subjetivo, no puede acusar a la persona de una negligencia o cosas así, sino que simplemente describir la situación de una manera comparando como fue la situación y como se debiera haber hecho esa evaluación formativa"

7. Muestra empatía con el - "si usted quiere ser un buen supervisor tiene que estar en contacto permanente con residente ese residente a lo largo de un tiempo"

- "... a mi me gustaría que mis residentes sientan que sé quiénes son ellos, que yo no solamente sea el nombre y el apellido sino que sea algo más de ellos, que hayamos tenido el espacio informal en donde pudiéramos comunicarnos como personas y esos espacios informales son complejos por los tiempos" 
8. Estimula la autonomía decisional progresiva en los residentes
- "el alumno en la medida que va avanzando en el programa va adquiriendo una responsabilidad directa sobre el manejo del enfermo que va a ser cada vez mayor"

- "uno de los elementos que uno debe estimular mucho en el becado es la autogestión de su conocimiento, enseñarles a hacerse preguntas significativas sobre aquello que están enfrentado, un paciente, un problema clínico, etc... y buscar respuestas que tengan significado y que sirvan para tomar decisiones respecto de un problema y que tengan solidez"

"...y también darle su espacio para que hagan esta autogestión y busquen pero siempre estar disponible para que puedan resolver sus dudas con uno o sin uno"

la educación continua

10. Establece prioridades asistenciales/docentes en situación de estrés

- "eso es lo que hace la mejor supervisión y no es un asunto fundamentalmente cuantitativo de cuántos enfermos va a ver en la mañana, sino que es más bien un aspecto cualitativo, y basta con que se haga muy bien en un par enfermos que estén en la parte más aguda..."

11. Trabaja en equipo

- "eso es fundamental usted tiene que incorporar oportunamente a la enfermera, al kinesiólogo, usted tiene que incorporarla oportunamente con respeto y con la capacidad de explicación de lo que debe hacer que esa persona se sienta tan profesional como usted"

- "en nuestro grupo, que manejamos pacientes oncológicos, las grandes decisiones son de equipo"

12. Ajusta la docencia a heterogeneidad y variabilidad de los grupos de estudiantes pre/postgrado

- "el mercado de la formación de postgrado es bien complejo, han crecido los grupos, la heterogeneidad de las personas, los modelos también son distintos al interior de nuestro grupo de gente"

3. Evalúa en forma objetiva, coherente y diversa en función de objetivos

14. Destreza en la ejecución de procedimientos simples y complejos en contacto con pacientes

15. Emplea casos derivados de la carga asistencial para el aprendizaje propio, de los colegas y residentes

16. Muestra respeto por el residente como colega

- "sería importante que se le diga a los residentes cuál es el programa de formación, qué es lo que se pretende como objetivos de aprendizaje, que supieran qué es lo que se va a evaluar..."

- "Conocimiento de la patología que está a cargo de la especialidad de uno, es una cosa básica, los elementos básicos del examen para poder llegar a un diagnóstico y un tratamiento"

- "...eso es lo que se llama reunión clínica generalmente se hace en un auditorio, se le encarga a un residente bajo la dirección de un supervisor, la reunión se parte con la exposición del caso y se sigue el hecho histórico de cuando se encontró la enfermedad, cuales son los síntomas... indudablemente que esas reuniones tienen valor, pero como le dije la actitud del asistente del que asiste a eso es totalmente distinta porque oye el cuento pero no lo vive, no lo sufre"

- "...que no se sienta aplastado el residente por el supervisor sino que lo sienta como un colaborador, como un amigo"

- "difícilmente ellos pueden humanizarse si están en un sistema donde ellos mismos son infrahumanos"

17. Transmite una "manera de pensar", de enfrentar el diagnóstico y el tratamiento para cada paciente

- "...y lo último es el poder entregar, y esto se hace sobre todo a través del modelaje y a través del feedback, componentes que tienen que ver con las actitudes"

- "yo creo que es súper interesante lo del conocimiento pero también lo de las estrategias para aproximarse al problema"

18. Muestra disponibilidad para - "...que los supervisores vayan cuando los llaman, estén ahí cuando a la hora que sea el residente en turno necesario, vean al enfermo, examinen al enfermo con el residente... el resultado para el enfermo no tiene comparación alguna"

19. Muestra apertura a recibir preguntas y enseña en forma no amenazante a aprender de errores propios y ajenos
- "uno va aprendiendo de los errores y es una cosa que uno les está enseñando" - "yo creo que la cercanía es importante, crea un clima grato, no se sienten amenazados..." 
20. Modela una relación médico- - "el supervisor debe ser entrenado no sólo en su técnica médica, en su especialidad, sino paciente en la entrevista que también en todas estas disciplinas que corren paralelamente de la relación humana clínica y que son tan importantes"

- "hay que buscar maneras y espacios para que los becados nos vean hacer cosas, para nosotros va como en lo intuitivo, pero muchas veces los mandamos a hacer cuestiones y yo resuelvo algunas partes del problema"

21. Fomenta la autoprotección - " "y una cosa importante es la dimensión humana al acercarse al enfermo y este tema en en el ejercicio de la profesión médica, frente al dolor propio, al de los pacientes $y$ el cáncer es re potente. Y lo otro es enseñarles a defenderse ellos"

- "los elementos más importantes que son lo cognitivo, las habilidades, y en un nivel más arriba está el aprender a aprender y aprender a cuidarse" su familia

22. Muestra el sistema público de salud como una instancia "Hay un rol de motivación al servicio público" valiosa de ejercicio de la especialidad

23. Genera la posibilidad de mantener intercambio profesional una vez finalizada la formación - "Nosotros lo hacemos, nuestros ex becados que se han ido a provincia mandan sus
casos con sus presentaciones... nosotros les mandamos nuestra opinión. Es súper fácil
hacer eso hoy en día"

- "algunas personas hacen eso de una forma casi instintiva... otras personas simplemente lo ignoran, y otras personas lo hacen con poca discreción, con mal criterio haciendo preguntas que no se deben hacer" paciente

- "hay docentes que no sólo lo promueven sino que le piden al residente de $3^{\text {er }}$ año que lo sustituya a él, sí, y que le haga el trabajo" aprendizaje entre pares

26. Promueve rol docente en los residentes
- "yo creo que vale la pena que el alumno aprenda enseñando, pero hay que tener muy en cuenta que los alumnos que se están formando como especialistas no tienen en la gran mayoría de los casos vocación docente" tenecían a 66 rotaciones clínicas; $87 \%$ en centros de nuestra institución y $13 \%$ en hospitales públicos. De los docentes evaluados, $4,1 \%$ era profesor titular, $17,1 \%$ profesor asociado, 33,3\% profesor asistente, $13,8 \%$ instructor y $22 \%$ no tenía grado académico.

La encuesta y su estadística descriptiva se muestran en la Tabla 2. El porcentaje de respuesta de los ítems fue entre 94 y 100\%, con una media entre 4,16 y 4,85 (Escala Likert 1-5). El puntaje global (14 ítems) fue 4,46 $\pm 0,94$ (89\% del máximo) y la nota final promedio fue de 6,42 $\pm 0,96$ en escala 1 a 7 ( $92 \%$ del máximo).

\section{Validez del constructo}

El análisis factorial mostró que el instrumento MEDUC-PG14 posee una estructura de 3 factores, de acuerdo al criterio de auto-valor (eigenvalue) $>1$ (Figura 2). El primer factor dio cuenta de $56 \%$ de la varianza, mientras que los 3 factores en conjunto explicaron $75,48 \%$ de la varianza total.
El primer factor fue Enseñanza y evaluación (EE), el cual contiene los ítems 1, 2, 3, 4, 8, 9, 13 y 14. El segundo, denominado Trato respetuoso a pacientes y equipo de salud (TR), contiene los ítems 5, 6 y 7. Finalmente, el tercero, denominado Entrega de retroalimentación (ER), incluye los ítems 10, 11 y 12.

En cuanto a los promedios globales, el dominio mejor evaluado se relacionó al trato respetuoso con 4,85 \pm 0,42 (97\% del máximo), seguido por el dominio relacionado a la enseñanza y evaluación con 4,45 \pm 0,82 (89\% del máximo), y finalmente hubo más aspectos por mejorar en la entrega de retroalimentación con 4,09 $\pm 1,0$ (82\% del máximo) (Tabla 3).

\section{Análisis de confiabilidad}

El instrumento MEDUC-PG14 obtuvo un alfa de Cronbach global de 0,947 (máximo de 1), lo que refleja una alta confiabilidad. Además, el coeficiente $\alpha$ de Cronbach para de los factores EE, TR y ER fue $0,96,0,88$ y 0,76 respectivamente (Tabla 4 ). 
Tabla 2. Cuestionario final de 14 ítems para la evaluación docente en postgrado (MEDUC-PG14)

\begin{tabular}{|lcc|}
\hline ITEMS & $\begin{array}{c}\text { Porcentaje } \\
\text { de respuestas }\end{array}$ & Media* y DE \\
\hline 1. Facilitó el proceso de enseñanza y aprendizaje de conocimientos de la especialidad & 100 & $4,59 \pm 0,69$ \\
2. Incorporó metodología de enseñanza eficiente y motivadora & 100 & $4,25 \pm 1,02$ \\
\hline 3. Dedicó el suficiente tiempo para enseñar y recalcar conceptos importantes & 99 & $4,30 \pm 0,99$ \\
\hline 4. Estuvo disponible y dedicó el tiempo necesario para la supervisión & 100 & $4,47 \pm 0,88$ \\
\hline 5. Demostró un trato respetuoso hacia los residentes & 100 & $4,85 \pm 0,53$ \\
\hline 6. Demostró un trato respetuoso con el equipo de trabajo (médicos, enfermeras, & 100 & $4,85 \pm 0,44$ \\
\hline auxiliares, kinesiólogos) & 100 & $4,87 \pm 0,43$ \\
\hline 7. Demostró un trato empático y respetuoso con los pacientes y su familia & 98 & $4,41 \pm 0,81$ \\
\hline $\begin{array}{l}\text { 8. Lo evaluó respecto a su conocimiento y destrezas clínicas } \\
\text { 9. Promovió la realización de procedimientos de acuerdo al nivel de conocimientos }\end{array}$ & 94 & $4,49 \pm 0,92$ \\
\hline y competencias adquirido por Ud. & 97 & $3,75 \pm 1,48$ \\
\hline 10. Realizó feedback durante esta rotación destacando sus fortalezas y aspectos por \\
mejorar (al menos 1 vez formalmente)
\end{tabular}

*Escala Likert 1 a 5. DE: desviación estándar.

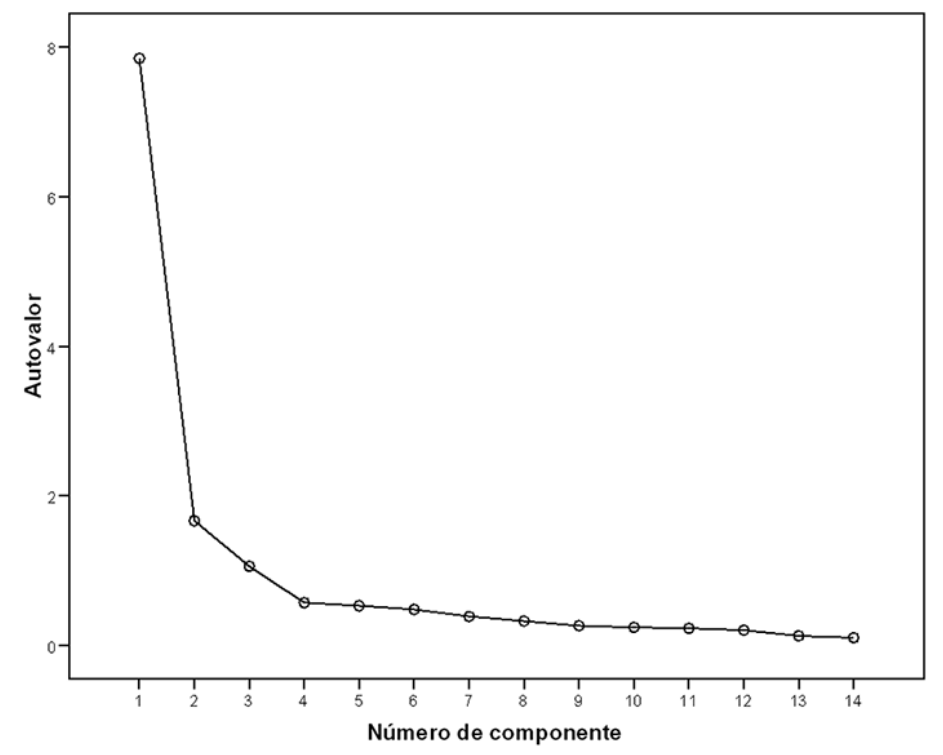

Figura 2. Gráfico de sedimentación: El eje $Y$ representa al autovalor en función del número de factores o componentes (eje X). 
Tabla 3. Resultados según dominio de la encuesta MEDUC-PG14

\begin{tabular}{|lcc|}
\hline Dominios & \multicolumn{1}{|c|}{ ítems } & Media y DE \\
\hline 1. Enseñanza y evaluación (8 ítems) & $1,2,3,4,8,9,13,14$ & $4,45 \pm 0,82$ \\
\hline 2. Trato respetuoso a pacientes y equipo de salud (3 ítems) & $5,6,7$ & $4,85 \pm 0,42$ \\
\hline 3. Entrega de retroalimentación (3 ítems) & $10,11,12$ & $4,09 \pm 1,0$ \\
\hline \multicolumn{2}{l}{ Calificación global (14 ítems) } & $4,46 \pm 0,94$ \\
\hline
\end{tabular}

Tabla 4. Confiabilidad del instrumento MEDUC-PG14

\begin{tabular}{|c|c|}
\hline Dominio & \multirow{2}{*}{$\begin{array}{c}\begin{array}{c}\text { Alfa de } \\
\text { Cronbach }\end{array} \\
0,956\end{array}$} \\
\hline 1. Enseñanza y evaluación & \\
\hline $\begin{array}{l}\text { 2. Trato respetuoso a pacientes y equipo } \\
\text { de salud }\end{array}$ & 0,875 \\
\hline 3. Entrega de retroalimentación & 0,760 \\
\hline Global & 0,947 \\
\hline
\end{tabular}

\section{Discusión}

Este estudio abordó los aspectos metodológicos vinculados a la creación y evaluación de las propiedades psicométricas del instrumento MEDUC-PG14, desarrollado para calificar el desempeño docente en programas de especialidades médicas. Fue piloteado en 12 especialidades, tanto pediátricas como del adulto, las que abarcaron un amplio espectro programas de formación.

Dado que se obtuvo información de múltiples fuentes involucradas en el proceso educativo en postgrado (mediante metodología mixta, cualitativa y cuantitativa), basada en un modelo teórico validado, consideramos que MEDUC-PG14 posee una alta validez de contenido, con alta concordancia entre las categorías emergentes del análisis cualitativo ${ }^{18}$, la posterior triangulación de la información ${ }^{20}$ y el contenido final de los ítems, refinados por el panel de expertos. Si consideramos la participación de residentes, docentes de diversos programas de postítulo y un representante de APICE, podemos inferir que se trata de un instrumento de validez nacional.

La validez de constructo fue realizada utilizando un análisis factorial exploratorio, seguido de una rotación Varimax. Los factores se seleccio- naron utilizando los criterios de Kaiser-Guttman y Cattell. De acuerdo a éstos, el análisis sugirió un instrumento multidimensional de tres categorías $^{16,25}$.

Por otra parte, el análisis de confiabilidad muestra que MEDUC-PG14 es altamente confiable, ya que posee un alfa de Cronbach 0,947. Además, los 3 dominios poseen un alfa de Cronbach $>0,7$, demostrando una buena confiabilidad de cada factor por separado ${ }^{27}$.

El cuestionario MEDUC-PG14 es fácil de aplicar (menos de $5 \mathrm{~min}$ ) y no requiere entrenamiento previo para realizarlo, constituyendo una fortaleza adicional, ya que mejora su aceptación entre residentes y jefes de programas, impactando positivamente en la tasa de respuesta al final de cada rotación ${ }^{27}$. Los resultados de MEDUC-PG14 demostraron que la mayor fortaleza de los docentes fue el trato respetuoso, mientras que hay otros aspectos por mejorar vinculados a la entrega de retroalimentación $(\text { feedback })^{10}$. Pese a que este dominio fue el más bajo, obtuvo una buena calificación ( $82 \%$ del máximo) y constituye una mejora respecto a evaluaciones de docentes de pregrado en la misma institución ${ }^{13}$. Además, la calificación global fue $89 \%$ del máximo, lo que refleja una buena percepción general de los residentes sobre el desempeño de sus profesores.

Cabe destacar que un porcentaje importante de los residentes respondió no aplica en el ítem 9 (37\% de respuestas), debido a que este mide específicamente la realización de procedimientos prácticos, los que no son habituales en algunas especialidades. Esto no se observó en otros ítems, ya que son habilidades docentes transversales a las especialidades médicas. Pese a la baja tasa de respuesta, la información obtenida es valiosa para aquellos docentes/rotaciones que incluyen la realización o supervisión de procedimientos y por otro lado, la baja tasa de respuesta no tuvo un impacto 
negativo en la confiabilidad del instrumento por lo que el equipo de investigación decidió mantenerlo en el instrumento definitivo ${ }^{26}$.

La capacitación de los jefes de programa es un aspecto trascendental para perfeccionar los programas de especialidad. Sobre esta capacitación, la retroalimentación (entrega y recepción) es considerada una necesidad importante a incluir ${ }^{26}$. En relación a esto, MEDUC-PG14 permitirá a los jefes de programa conocer el desempeño de sus docentes y entregarles retroalimentación estructurada y focalizada en relación a sus fortalezas y debilidades. También ayudará a realizar estrategias destinadas a mejorar las habilidades en dichos aspectos.

Los programas de capacitación docentes son fundamentales para mejorar la docencia en una facultad de medicina ${ }^{5,7}$. Tras su implementación en nuestro centro, se observaron cambios tanto en el desempeño del profesor, como también en su desarrollo personal, su rol como médico, en la comunidad académica y en la institución a la que pertenece ${ }^{28-31}$. Consideramos que MEDUC-PG14 contribuirá a la planificación y mejora de los programas de capacitación de docentes de postgrado. Asimismo, los resultados individuales podrían estimular a aquellos profesores que no han recibido capacitación docente a realizar cursos de docencia, mejorando así su desempeño individual y realización profesional ${ }^{32}$.

A partir de los resultados, se podrían realizar nuevos estudios para conocer otras propiedades del instrumento, como el estudio D (teoría de la generalización) o la capacidad de MEDUC-PG14 para detectar a los mejores docentes, promoviendo la excelencia entre los profesores clínicos y una vía de promoción académica al interior de una institución. Otro desafío sería la evaluación a futuro, de la validez y confiabilidad del instrumento en otras escuelas de postgrado a nivel nacional e internacional.

Finalmente, MEDUC-PG14 es un instrumento multidimensional, válido y muy confiable para evaluar docentes en programas de especialidades médicas. Además, proporciona retroalimentación para mejorar la calidad docente de nuestros profesores y constituye el primer instrumento en español validado para postgrado en América Latina.

Agradecimientos: Artículo financiado por el Fondo Nacional de Desarrollo Científico y Tecnológico (FONDECYT) proyectos $\mathrm{N}^{\circ} 1120652$
(A.R.) y 1120534 (M.B.) Agradecemos la valiosa colaboración de la Dirección de Postgrado de la Pontificia Universidad Católica de Chile y de la Agencia Acreditadora de Programas de Postgrado, de Especialidad en Medicina y de Centros Formadores de Especialistas Médicos (APICE).

\section{Referencias}

1. Robin BR, McNeil SG, Cook DA, Agarwal KL, Singhal GR. Preparing for the changing role of instructional technologies in medical education. Acad Med 2011; 86: 435-9.

2. Davis DA, Prescott J, Fordis CM Jr, Greenberg SB, Dewey CM, Brigham T, et al. Rethinking CME: an imperative for academic medicine and faculty development. Acad Med 2011; 86: 468-73.

3. Holmboe ES, Ward DS, Reznick RK, Katsufrakis PJ, Leslie KM, Patel VL, et al. Faculty development in assessment: the missing link in competency-based medical education. Acad Med 2011; 86: 460-7.

4. Amin Z, Hoon Eng K, Gwee M, Chay Hoon T, Dow Rhoon $\mathrm{K}$. Addressing the needs and priorities of medical teachers through a collaborative intensive faculty development programme. Med Teach 2006; 28: 85-8.

5. Trivino X, Sirhan M, Moore P, Reyes C. [Faculty development for clinical teachers in medicine]. Rev Med Chile 2009; 137: 1516-22.

6. O'Sullivan PS, Irby DM. Reframing research on faculty development. Acad Med 2011; 86: 421-8.

7. Steinert Y. Faculty development in the new millennium: key challenges and future directions. Medical Teacher 2000; 22: 44-50.

8. Benor DE. Faculty development, teacher trainning and teacher accreditation in medical education: Twenty years from now. Medical Teacher 2000; 22: 503-12.

9. Castanelli D, Kitto S. Perceptions, attitudes, and beliefs of staff anaesthetists related to multi-source feedback used for their performance appraisal. British Journal of Anaesthesia 2011; 107 (3): 372-7.

10. Hattie J, Timperley H. The Power of Feedback. Review of Educational Research 2007; 77: 81-112.

11. Schartel SA. Giving feedback-An integral part of education. In: Best practice \&amp; research Clinical anaesthesiology: Elsevier Ltd; 2012. p. 77-87.

12. Stalmeijer RE, Dolmans DH, Wolfhagen IH, Muijtjens AM, Scherpbier AJ. The Maastricht Clinical Teaching Questionnaire (MCTQ) as a valid and reliable instrument for the evaluation of clinical teachers. Acad Med 2010; 85: 1732-8. 
13. Bitran M, Mena B, Riquelme A, Padilla O, Sánchez I, Moreno R. [An instrument in Spanish to evaluate the performance of clinical teachers by students]. Rev Med Chile 2010; 138: 685-93.

14. Huete GÁ, Julio GR, Rojas DV, Herrera RC, Padilla PO, Solis LN, et al. Desarrollo y validación del instrumento MEDUC-RX32, para la evaluación de docentes de programas de la especialidad de postítulo en radiología. Revista Chilena de Radiología 2014; 20: 75-80.

15. Zuberi RW, Bordage G, Norman GR. Validation of the SETOC instrument-Student evaluation of teaching in outpatient clinics. Adv Health Sci Educ Theory Pract 2007; 12: 55-69.

16. Litzelman DK, Stratos GA, Marriott DJ, Skeff KM. Factorial validation of a widely disseminated educational framework for evaluating clinical teachers. Acad Med 1998; 73: 688-95.

17. Williams BC, Litzelman DK, Babbott SF, Lubitz RM, Hofer TP. Validation of a global measure of faculty's clinical teaching performance. Acad Med 2002; 77: 177-80.

18. Corbin JM, Strauss A. Grounded theory research: Procedures, canons, and evaluative criteria. Qualitative sociology 1990; 13: 3-21.

19 O'Cathain A, Murphy E, Nicholl J. Three techniques for integrating data in mixed methods studies. BMJ 2010; 341: c4587.

20. Moran-Ellis J, Alexander VD, Cronin A, Dickinson M, Fielding J, Sleney J, et al. Triangulation and integration: processes, claims and implications. Qualitative Research 2006; 6: 45-59.

21. Kaiser $H$. The Varimax criterion for analytic rotation in factor analysis. Psychometrika 1958; 23: 187-200.

22. Field A. Discovering statistics using SPSS for Windows (Second Edition). London: Sage Publications Limited, 2005.
23. Field A. Discovering statistics using SPSS for Windows. London: Sage Publications Limited, 2000.

24. Cattell RB. The scree test for a number of factors. Multivariate Behavioural Research 1966; 1: 245-76.

25. Cronbach L. Coefficient alpha and the internal structure of tests. Psychometrika 1951; 16: 297-334.

26. Herrera CA, Niklitschek I, Pizarro M, Solís N, Olivos $\mathrm{T}$, Rojas V, et al. Identificación de las necesidades de capacitación docente de los jefes de programa de especialización médica. Rev Med Chile 2013; 141: 1126-35.

27. Van Der Vleuten CPM. The assessment of professional competence: Developments, research and practical implications. Advances in Health Sciences Education 1996; 1: 41-67.

28. Trivino X, Sirhan M, Moore P, Montero L. [Impact of a diploma on medical education in a medical school in Chile]. Rev Med Chile 2011; 139: 1508-15.

29. Sirhan M, Trivino X. [Evaluation of a faculty development program in curriculum development for program directors of medical specialties]. Rev Med Chile 2012; 140: $530-7$

30. Moore P, Montero L, Trivino X, Sirhan M, Leiva L. [Impact beyond the objectives: a qualitative study of a faculty development program in medical education]. Rev Med Chile 2014; 142: 336-43.

31. Trivino X, Sirhan M, Moore P, Montero L. [Experiences in the implementation of a faculty development program for teching for professors of a university of medicine in Chile]. Rev Peru Med Exp Salud Publica 2014; 31: 417-23.

32. Montero L, Trivino X, Sirhan M, Moore P, Leiva L. [Barriers for faculty development in medical education: a qualitative study]. Rev Med Chile 2012; 140: 695-702. 